\title{
Eine konkrete Menge der Kardinalität Aleph-1
}

\author{
Gerald Kuba \\ Gerald Kuba schloß 1985 sein Mathematikstudium an der Universität Wien ab, um \\ danach als wissenschaftlicher Mitarbeiter an der wirtschaftstheoretischen Fakultät der \\ Universität Bielefeld tätig zu sein. Er promovierte 1991 an der Universität Wien und ist \\ seit 2001 Dozent für Mathematik ebendort, sowie außerordentlicher Professor an der \\ Universität für Bodenkultur Wien, wo er seit 1994 beschäftigt ist. Sein wissenschaftli- \\ ches Hauptinteresse gilt der analytischen Zahlentheorie, speziell der Gitterpunktlehre.
}

\section{Einleitung}

Eine unendliche Menge $\mathcal{A}$ heißt abzählbar bzw. von der Kardinalität $\aleph_{0}$, wenn $\mathcal{A}$ gleichmächtig mit der Menge $\mathbb{N}$ der natürlichen Zahlen ist. (Bekanntlich nennt man zwei Mengen $X$ und $Y$ gleichmächtig, wenn $X$ bijektiv auf $Y$ abgebildet werden kann.) Einfache Beispiele von Mengen der Kardinalität $\aleph_{0}$ sind alle unendlichen Teilmengen der Menge $\mathbb{Q}$ der rationalen Zahlen, insbesondere die Mengen $\mathbb{N}$ und $\mathbb{Q}$. Unendliche Mengen, die nicht abzählbar sind, nennt man überabzählbar. (Da jede unendliche Menge eine Teilmenge der Kardinalität $\aleph_{0}$ enthält, ist eine überabzählbare Menge stets ,größer“ als eine abzählbare.) Das wichtigste Beispiel einer überabzählbaren Menge ist die Zahlengerade $\mathbb{R}$. Da die Menge $\mathbb{R}$ gleichmächtig mit der Potenzmenge von $\mathbb{N}$ ist, ordnet man $\mathbb{R}$ die

In der Hierarchie des Unendlichen ist $\aleph_{0}$ die kleinste Kardinalzahl und wird allen abzählbar unendlichen Mengen zugewiesen. Die nächstgrößere Kardinalzahl nach $\aleph_{0}$ ist $\aleph_{1}$. Der Prototyp einer Menge der Kardinalität $\aleph_{1}$ ist die sog. zweite Zahlklasse, die für die Anwendung der ordinalen Mengenlehre in der Topologie und der Maßtheorie von großer Bedeutung ist. Diese Menge wird mit Hilfe der abzählbaren Ordinalzahlen bzw. Wohlordnungstypen gebildet. Grundidee dieser Vorgehensweise ist es, verschiedene Ordnungen auf einer festen Menge zu betrachten. In der hier vorgestellten Konstruktion einer Menge der Kardinalität $\aleph_{1}$ wird dagegen eine natürliche Universalordnung auf verschiedenen Mengen verwendet. Dadurch läßt sich das Ganze in der allgemein vertrauteren Sprache der Analysis erklären; sowohl das Konzept der transfiniten Ordinalzahl als auch die Begriffe Ordnungsisomorphie bzw. Ordnungstyp können somit vermieden werden. 
Kardinalzahl $2^{\aleph_{0}}$ zu und dokumentiert die Überabzählbarkeit von $\mathbb{R}$ durch die Relation $2^{\aleph_{0}}>\aleph_{0}$. (Mit CANTORs unsterblichem Beweis dieser Relation im Dezember 1873 schlug die Geburtsstunde der Mengenlehre.)

Eine Menge $\mathcal{M}$ heißt von der Kardinalität $\aleph_{1}$, wenn $\mathcal{M}$ überabzählbar ist und jede unendliche Teilmenge von $\mathcal{M}$ entweder von der Kardinalität $\aleph_{0}$ oder gleichmächtig mit $\mathcal{M}$ selbst ist. Selbstverständlich sind zwei Mengen der Kardinalität $\aleph_{1}$ stets gleichmächtig, sodaß $\aleph_{1}$ die nächstgrößere Kardinalzahl nach $\aleph_{0}$ darstellt und somit $\aleph_{0}<\aleph_{1} \leq 2 \aleph^{\aleph_{0}}$ gilt. Die (spezielle) Kontinuumshypothese besagt, daß $\aleph_{1}=2^{\aleph_{0}}$ gilt. Unter dieser Voraussetzung ist die Zahlengerade $\mathbb{R}$ ein anschauliches Beispiel einer Menge der Kardinalität $\aleph_{1}$. Allerdings ist nach den berühmten Sätzen von GöDEL und COHEN die Kontinuumshypothese im Rahmen einer axiomatischen Standardmengenlehre weder beweisbar noch widerlegbar.

Unabhängig von der Kontinuumshypothese enthält $\mathbb{R}$ zwar unendlich viele Teilmengen der Kardinalität $\aleph_{1}$, aber ohne Kontinuumshypothese läßt sich keine dieser Mengen konkret realisieren. Tatsächlich erweist es sich als gar nicht so einfach, auch nur irgendeine Menge der Kardinalität $\aleph_{1}$ konkret anzugeben. Um das zu erreichen, wird in der Literatur stets mit einem starken Instrumentarium aus ordinaler Mengenlehre hantiert. Dieses ist zwar zum Studium der Mengenlehre unverzichtbar, zur Konstruktion eines ,anschaulichen“ Beispiels einer Menge der Kardinalität $\aleph_{1}$ kann man aber einen wesentlich einfacheren Weg beschreiten, der unnötige Ausflüge in die ordinale Mengenlehre vermeidet.

\section{Die Konstruktion}

Ausgangspunkt unserer Konstruktion ist die (abzählbare) Menge $\mathbb{Q}_{+}$der nichtnegativen rationalen Zahlen zusammen mit ihrer natürlichen Ordnung $\leq$. Eine Teilmenge $A$ von $\mathbb{Q}_{+}$nennen wir wohlgeordnet, wenn jede nichtleere Teilmenge $T$ von $A$ ein kleinstes Element enthält. (Dieses eindeutig bestimmte Element bezeichnen wir wie üblich mit $\min T$.) Speziell ist $\varnothing$ wohlgeordnet, während natürlich $\mathbb{Q}_{+}$nicht wohlgeordnet ist. Nun sei $\mathcal{W}$ die Menge aller wohlgeordneten Teilmengen von $\mathbb{Q}_{+}$. Zur Illustration führen wir an: $\mathbb{N} \in \mathcal{W}$, sowie $N_{2} \in \mathcal{W}$ mit $N_{2}:=\left\{1-\frac{1}{n+1} \mid n \in \mathbb{N}\right\} \cup\left\{2-\frac{1}{n+1} \mid n \in \mathbb{N}\right\}$. Nebenbei erwähnt ist es klar, daß endliche Vereinigungen und beliebige Durchschnitte von (mindestens zwei) Mengen in $\mathcal{W}$ wieder in $\mathcal{W}$ liegen. In der Menge $\mathcal{W}$ haben wir allerdings noch nicht unsere Menge $\mathcal{M}$ der Kardinalität $\aleph_{1}$ gefunden, da wegen $T \in \mathcal{W}$ für alle $T \subset \mathbb{N}$ die Menge $\mathcal{W}$ gleichmächtig mit $\mathbb{R}$ ist, wir aber die Kontinuumshypothese nicht voraussetzen wollen. Wir erhalten unsere Menge $\mathcal{M}$ durch eine zweckmäßige Verkleinerung der Menge $\mathcal{W}$. Konkret geschieht dies mit Hilfe einer Äquivalenzrelation. Wir nennen zwei Mengen $A, B \in \mathcal{W}$ äquivalent und schreiben $A \sim B$ genau dann, wenn es eine monoton wachsende Bijektion von $A$ nach $B$ gibt. Es ist klar, daß damit tatsächlich eine Äquivalenzrelation auf $\mathcal{W}$ definiert wird. Zur Illustration stellen wir fest, daß für eine unendliche Teilmenge $T$ von $\mathbb{N}$ stets $T \sim \mathbb{N}$ gilt, daß aber z.B. die oben angeführte Menge $N_{2}$ offensichtlich nicht mit $\mathbb{N}$ äquivalent ist. Abkürzend bezeichnen wir für einen Repräsentanten $A \in \mathcal{W}$ mit $[A]$ die Äquivalenzklasse $\{B \in \mathcal{W} \mid B \sim A\}$. Unsere Menge $\mathcal{M}$ entsteht nun aus $\mathcal{W}$, indem man zur Quotientenmenge $\mathcal{W} / \sim$ übergeht. Wir setzen also schließlich $\mathcal{M}:=\{[A] \mid A \in \mathcal{W}\}$ und behaupten:

Satz. Die Menge $\mathcal{M}$ besitzt die Kardinalität $\aleph_{1}$. 


\section{Vorbereitung des Beweises}

Zunächst stellen wir fest, daß $\mathcal{M}$ eine unendliche Menge darstellt. Denn sie enthält jedenfalls die Äquivalenzklassen aller endlichen Teilmengen von $\mathbb{Q}_{+}$und zwei endliche Teilmengen von $\mathbb{Q}_{+}$sind offensichtlich genau dann äquivalent, wenn sie gleich viele Elemente enthalten. Den eigentlichen Beweis des Satzes zerlegen wir in zwei Teile:

Satz 1 Die Menge $\mathcal{M}$ ist überabzählbar.

Satz 2 Jede überabzählbare Teilmenge $\mathcal{D}$ von $\mathcal{M}$ muß mit $\mathcal{M}$ gleichmächtig sein.

Zum Beweis der beiden Sätze ist unterschiedliche Vorarbeit vonnöten. Natürlich ist der Beweis von Satz 2 aufwendiger als der von Satz 1, den wir gleich im Anschluß an das folgende Lemma erledigen können.

Lemma 1 Wenn $A \in \mathcal{W}$ und $r \in \mathbb{Q}_{+}$und $a \in A$, dann ist die (wohlgeordnete) Menge $A \cap[r, a[$ niemals äquivalent mit $A$.

Beweis. Angenommen, es gibt eine monoton wachsende Bijektion $f: A \rightarrow A \cap[r, a[$. Dann ist die Umkehrfunktion $g=f^{-1}$ natürlich ebenfalls monoton wachsend. Ein scharfer Blick auf die Mengen $\{x \in A \mid x>f(x)\}$ und $\{y \in A \cap[r, a[\mid y>g(y)\}$ lehrt einen, daß beide Mengen leer sein müssen, da sie wegen der strengen Monotonie von $f$ bzw. $g$ kein Minimum besitzen können. Es gilt also $x \leq f(x)$ für alle $x \in A$ und $y \leq g(y)$ für alle $y \in A \cap[r, a[$. Ist nun $x \in A$, so ist also einerseits $x \leq f(x)$, andererseits ist $f(x) \leq g(f(x))=x$. Somit gilt $f(x)=x$ für alle $x \in A$ und das steht im Widerspruch $\mathrm{zu} f(a)<a$.

\section{Beweis von Satz 1}

Wir zeigen, daß, wenn immer $\mathcal{A}$ eine abzählbar unendliche Teilmenge von $\mathcal{M}$ ist, dann garantiert ein Element aus $\mathcal{M}$ in $\mathcal{A}$ fehlt. Es sei also $\mathcal{A}=\left\{\left[A_{n}\right] \mid n \in \mathbb{N}\right\} \subset \mathcal{M}$ mit irgendwelchen paarweise nicht äquivalenten Repräsentanten $A_{n} \in \mathcal{W}$. Nun betrachten wir für jedes $n \in \mathbb{N}$ die auf $\mathbb{Q}_{+}$definierte streng monoton wachsende Funktion $f_{n}$, die durch $f_{n}(x)=n+\frac{x}{1+x}$ gegeben ist. Für jedes $n \in \mathbb{N}$ ist dann die Menge $A_{n}$ äquivalent mit dem Bild $f_{n}\left(A_{n}\right)$ von $A_{n}$ unter $f_{n}$, das eine Teilmenge des Intervalls [ $n, n+1$ [ darstellt. Wir können daher bei der Wahl der Repräsentanten $A_{n}$ gleich vorweg $A_{n} \subset[n, n+1[$ annehmen, sodaß die Repräsentanten paarweise disjunkt sind und für $n<m$ stets $x<y$ für alle $x \in A_{n}$ und $y \in A_{m}$ gilt. Wir betrachten nun die Menge $A:=\bigcup_{n \in \mathbb{N}} A_{n}$ und behaupten, daß $A \in \mathcal{W}$ gilt und $A \sim A_{n}$ für alle $n \in \mathbb{N}$ falsch ist, sodaß also $[A] \in \mathcal{M} \backslash \mathcal{A}$ gilt.

Daß $A$ eine wohlgeordnete Teilmenge von $\mathbb{Q}_{+}$darstellt, ist leicht zu verifizieren. Wenn $\emptyset \neq T \subset A$, dann gilt $\emptyset \neq T=\bigcup_{n \in \mathbb{N}} T \cap A_{n}$ und somit $\min T=\min \left(T \cap A_{m}\right)$, wobei $m$ die kleinste natürliche Zahl mit $T \cap A_{m} \neq \emptyset$ ist. Daß $A$ nicht mit einer Menge $A_{n}$ äquivalent sein kann, folgt schließlich aus Lemma 1 , da für alle $n \in \mathbb{N}$ offensichtlich $A_{n}=A \cap\left[\min A_{n}, \min A_{n+1}[\right.$ gilt. 


\section{Segmente}

Ist $A \in \mathcal{W}$, so nennen wir $A$ selbst, sowie jede Teilmenge von $A \operatorname{der}$ Form $A \cap[0, r[=$ $\{a \in A \mid a<r\}$ mit irgendeinem $r \in \mathbb{Q}_{+}$ein Segment von $A$. Ein Segment von $A$ ist also ein Intervall in $A$, das min $A$ enthält oder leer ist. Zur Abkürzung betrachten wir ferner die Menge $\mathcal{S}(A)$ aller Segmente von $A$ :

$$
\mathcal{S}(A):=\left\{A \cap \left[0, x\left[\mid x \in \mathbb{Q}_{+} \cup\{\infty\}\right\} \quad(A \in \mathcal{W}) .\right.\right.
$$

Es ist klar, daß $\mathcal{S}(A)$ für jedes $A \in \mathcal{W}$ eine abzählbare Teilmenge von $\mathcal{W}$ ist. Ferner gilt für $S_{1}, S_{2} \in \mathcal{S}(A)$ stets $S_{1} \subset S_{2}$ oder $S_{2} \subset S_{1}$. Schließlich ist $\mathcal{S}(S)$ stets eine Teilmenge von $\mathcal{S}(A)$ für $S \in \mathcal{S}(A)$.

Lemma 2 Zwei verschiedene Segmente von $A \in \mathcal{W}$ sind niemals äquivalent.

Beweis. Sind $S_{1}$ und $S_{2}$ zwei verschiedene Segmente von $A$, so sei o.B.d.A. $S_{1} \subset S_{2}$, sodaß wir $S_{1}=A \cap\left[0, x_{1}\left[\right.\right.$ und $S_{2}=A \cap\left[0, x_{2}\left[\right.\right.$ mit $x_{1}, x_{2} \in \mathbb{Q}_{+} \cup\{\infty\}$ und $x_{1}<x_{2}$ schreiben können. Wegen $A \cap\left[x_{1}, x_{2}\left[=S_{2} \backslash S_{1} \neq \varnothing\right.\right.$ können wir das Minimum $a$ von $S_{2} \backslash S_{1}$ hernehmen und dann gilt offensichtlich $S_{1}=S_{2} \cap\left[0, a\right.$ [. Daher können $S_{1}$ und $S_{2}$ nach Lemma 1 nicht äquivalent sein.

Nun betrachten wir für $A \in \mathcal{W}$ die Menge $\Sigma[A]:=\{[S] \mid S \in \mathcal{S}(A)\}$ und stellen gleich fest, daß $\Sigma[A]$ eine Teilmenge von $\mathcal{M}$ darstellt, die nicht von $A$ sondern nur von der Äquivalenzklasse $[A]$ abhängt. Darüber hinaus gilt:

Lemma 3 Für $A, B \in \mathcal{W}$ gilt $\Sigma[A]=\Sigma[B]$ genau dann, wenn $A \sim B$ gilt.

Beweis. Zunächst zeigen wir, daß wenn $A \sim B$ gilt, dann $\Sigma[A] \subset \Sigma[B]$ gilt, was aus Symmetriegründen bereits mit $\Sigma[A]=\Sigma[B]$ gleichbedeutend ist. Es sei also $f: A \rightarrow$ $B$ bijektiv und monoton wachsend und $S$ ein Segment von $A$, also $[S]$ ein Element von $\Sigma[A]$. Da das Bild $f(S)$ von $S$ unter $f$ jedenfalls in $\mathcal{W}$ liegt und mit $S$ ähnlich ist, somit $[f(S)]=[S]$ gilt, genügt es zum Nachweis von $[S] \in \Sigma[B]$ zu zeigen, daß $f(S)$ ein Segment von $B$ ist. Das ist schnell erledigt: Im Falle $S=A$ haben wir $f(S)=B$ und nichts zu zeigen. Im Falle $S \neq A$ setzen wir $a=\min (A \backslash S)$ und bekommen damit ein $a \in A$ dergestalt, daß $S=A \cap[0, a$ [ gilt. Wegen der Monotonie von $f$ ist es klar, daß dann $f(S)=B \cap[0, f(a)[$ und somit $f(S) \in \mathcal{S}(B)$ gelten muß.

Nun nehmen wir umgekehrt an, daß $\Sigma[A]=\Sigma[B]$ gilt. Speziell gilt $[A] \in \Sigma[B]$ und $[B] \in \Sigma[A]$, d.h. es ist $A$ mit einem Segment von $B$ und $B$ mit einem Segment von $A$ ähnlich. Es gibt also $x, y \in \mathbb{Q}_{+} \cup\{\infty\}$ sowie monoton wachsende Bijektionen $f: A \rightarrow$ $B \cap[0, y[$ und $g: B \rightarrow A \cap[0, x[$. Falls $a<x$ für alle $a \in A$ oder $b<y$ für alle $b \in B$ gilt, sind wir fertig, da dann via $g$ oder via $f$ die Ähnlichkeit von $A$ und $B$ gewährleistet ist. Angenommen also, es gibt ein $a \in A$ mit $a \geq x$ und ein $b \in B$ mit $b \geq y$. Sind $a$ und $b$ minimal gewählt, dann gilt $A \cap[0, x[=A \cap[0, a[$ und $B \cap[0, y[=B \cap[0, b[$. Dann bildet die Funktion $g \circ f$ aber die Menge $A$ streng monoton wachsend auf die Menge $A \cap[0, a[\cap[0, g(b)[=A \cap[0, \min \{a, g(b)\}[\quad a b$. Diese beiden Mengen können nach Lemma 1 aber unmöglich äquivalent sein! 


\section{Beweis von Satz 2}

Als unmittelbare Folgerung von Lemma 3 erhalten wir

Korollar Durch $[A] \mapsto \Sigma[A]$ ist eine injektive Abbildung von $\mathcal{M}$ in die Potenzmenge von $\mathcal{M}$ definiert. Insbesondere ist die Menge

$$
\mathcal{M}^{*}:=\{\Sigma[A] \mid A \in \mathcal{W}\}
$$

von derselben Kardinalität wie die Menge $\mathcal{M}$.

Daher ist Satz 2 erledigt durch einen Beweis von

Satz 2' Jede überabzählbare Teilmenge $\mathcal{D}$ von $\mathcal{M}^{*}$ muß mit $\mathcal{M}^{*}$ gleichmächtig sein.

Dazu müssen wir die Menge $\mathcal{M}^{*}$ genauer unter die Lupe nehmen.

Lemma 4 Für alle $\mathcal{A}, \mathcal{B} \in \mathcal{M}^{*}$ gilt $\mathcal{A} \subset \mathcal{B}$ oder $\mathcal{B} \subset \mathcal{A}$.

Beweis. Sind $A, B \in \mathcal{W}$, so setzen wir $F=\{(a, b) \in A \times B \mid A \cap[0, a[\sim B \cap[0, b[\}$. Im Lichte von Lemma 3 ist durch $F$ eine Injektion definiert, die auf ihrem Definitionsbereich $\operatorname{dom} F$ monoton wachsend ist. Ferner stellen wir fest, daß dom $F$ ein Segment von $A$ ist. Tatsächlich gilt im Lichte des Beweises von Lemma $3 A \cap[0, a[\subset \operatorname{dom} F$ für alle $a \in \operatorname{dom} F$ und daher $\operatorname{dom} F=A \cap \bigcup_{a \in \operatorname{dom} F}[0, a]=A \cap\left[0, x\left[\right.\right.$ für ein $x \in \mathbb{Q}_{+} \cup\{\infty\}$.

Auf analoge Weise sieht man, daß auch das Bild im $F$ von $F$ ein Segment von $B$ ist. Da es ferner zu zwei äquivalenten Segmenten $S_{1} \in \mathcal{S}(A) \backslash\{A\}$ und $S_{2} \in \mathcal{S}(B) \backslash\{B\}$ stets äquivalente Segmente $S_{1}^{\prime}$ bzw. $S_{2}^{\prime}$ von $A$ bzw. $B$ gibt, die echte Obermengen von $S_{1}$ bzw. $S_{2}$ darstellen, ist es ausgeschlossen, daß sowohl dom $F \neq A$ als auch im $F \neq B$ gilt. Im Falle $\operatorname{dom} F=A$ ist via $F \quad A \sim \operatorname{im} F \in \mathcal{S}(B)$ und damit nach Lemma $3 \Sigma[A] \subset \Sigma[B]$ gewährleistet, während im Fall $\operatorname{im} F=B$ via $F^{-1} \quad B \sim \operatorname{dom} F \in \mathcal{S}(A)$ und damit $\Sigma[B] \subset \Sigma[A]$ gilt.

Der wesentliche Schritt zum Beweis von Satz 2' liegt nun in

Lemma 5 Für alle $\mathcal{A} \in \mathcal{M}^{*}$ ist die Menge $\left\{\mathcal{B} \in \mathcal{M}^{*} \mid \mathcal{B} \subset \mathcal{A}\right\}$ abzählbar.

Beweis. Nach dem vorigen Beweis ist es klar, daß für alle $A \in \mathcal{W}$

$$
\left\{\mathcal{B} \in \mathcal{M}^{*} \mid \mathcal{B} \subset \Sigma[A]\right\}=\{\Sigma[S] \mid S \in \mathcal{S}(A)\}
$$

gilt, sodaß damit also stets eine abzählbare Menge vorliegt.

Nun haben wir alles beieinander, um Satz $2^{\prime}$ zu beweisen. Es sei $\mathcal{D}$ eine überabzählbare Teilmenge von $\mathcal{M}^{*}$. Dann kann es wegen Lemma 5 kein $\mathcal{A} \in \mathcal{M}^{*}$ geben, sodaß $\mathcal{B} \subset \mathcal{A}$ für alle $\mathcal{B} \in \mathcal{D}$ gilt. Mit Lemma 4 gibt es somit zu jedem $\mathcal{A} \in \mathcal{M}^{*}$ ein $\mathcal{B} \in \mathcal{D}$ mit $\mathcal{B} \supset \mathcal{A}$. Daher können wir $\mathcal{M}^{*}=\bigcup_{\mathcal{B} \in \mathcal{D}}\left\{\mathcal{A} \in \mathcal{M}^{*} \mid \mathcal{A} \subset \mathcal{B}\right\}$ schreiben. Die überabzählbare Menge $\mathcal{M}^{*}$ ist auf diese Weise als Vereinigung von abzählbaren Mengen dargestellt. Die Indexmenge $\mathcal{D}$ dieser Vereinigung muß daher von der Kardinalität der Menge $\mathcal{M}^{*}$ sein. 


\section{Eine unzweckmäßige Variation}

Wir sind von $\mathbb{Q}_{+}$, einer Menge der Kardinalität $\aleph_{0}$, ausgegangen und haben mit $\mathcal{M}$ eine Menge der Kardinalität $\aleph_{1}$ gewonnen. Was passiert, wenn man das gleiche Spiel mit der Menge $\mathbb{R}_{+}$der nichtnegativen reellen Zahlen startet, die (so wie $\mathbb{R}$ ) von der Kardinalität $2^{\aleph_{0}}$ ist? Bekommt man dann eine Menge $\mathcal{M}$ der Kardinalität $\left(2^{\aleph_{0}}\right)^{+}$? (So wie $\aleph_{1}=\aleph_{0}^{+}$ die nächstgrößere Kardinalzahl nach $\aleph_{0}$ ist, soll $\kappa^{+}$die nächstgrößere Kardinalzahl nach $\kappa$ sein. Ist also eine Menge $K$ von der Kardinalität $\kappa$, dann ist $K^{+}$von der Kardinalität $\kappa^{+}$, wenn $K^{+}$gleichmächtig mit einer Obermenge $K^{\prime}$ von $K$ ist, die nicht gleichmächtig mit $K$ ist, sodaß aus $K \subset X \subset K^{\prime}$ stets folgt, daß $X$ entweder mit $K$ oder mit $K^{\prime}$ gleichmächtig ist.) Es ist auf den ersten Blick vielleicht verwunderlich, aber man bekommt nicht nur nicht eine Menge $\mathcal{M}$ der Kardinalität $\left(2^{\aleph_{0}}\right)^{+}$: Egal wie groß die Kardinalität $2^{\aleph_{0}}$ des Kontinuums tatsächlich ist, die Menge $\mathcal{M}$ hat, auch wenn man bei $\mathbb{R}_{+}$startet, wieder nur die Kardinalität $\aleph_{1}$ ! Der Grund dafür liegt darin, daß $\mathbb{R}_{+}$den wohlgeordneten Mengen nicht mehr Raum bieten kann als die in $\mathbb{R}_{+}$dicht liegende Menge $\mathbb{Q}_{+}$. Tatsächlich ist jede wohlgeordnete Teilmenge $A$ von $\mathbb{R}_{+}$zu einer Teilmenge $B$ von $\mathbb{Q}_{+}$äquivalent und damit insbesondere abzählbar. Man nehme etwa $B=\{\rho(a) \mid a \in A\}$ mit $\rho(a) \in$ $\mathbb{Q} \cap] a, \min (A \backslash[0, a])\left[\right.$ für $a \in A$ und $a \neq \max A$, sowie $\left.\rho\left(a^{\prime}\right) \in \mathbb{Q} \cap\right] a^{\prime}, \infty[$ im Falle, $\mathrm{da} ß a^{\prime}=\max A$ existiert.

\section{Gerald Kuba}

Institut für Mathematik

Universität für Bodenkultur

A-1180 Wien, Österreich

e-mail:kuba@edv1.boku.ac.at 